\title{
Loss Reduction of an ARROW Waveguide in Shorter Wavelength and Its Stack Configuration
}

\author{
T. BABA, Y. KOKUBUN, member, ieee, T. SAKAKI, and K. IGA, Fellow, IEeE
}

\begin{abstract}
We have reduced the propagation loss of an antiresonant reflecting optical waveguide (ARROW) to $0.3 \mathrm{~dB} / \mathrm{cm}$ in a short wavelength band by employing a transparent $\mathrm{TiO}_{2} / \mathrm{SiO}_{2}$ interference cladding. We developed a new analysis method for ARROW in order to analyze its propagation characteristics. In addition, we stacked two uncoupled parallel ARROW's with only $2-\mu \mathrm{m}$ spacing, aiming at threedimensional optical interconnection.
\end{abstract}

\section{INTRODUCTION}

A SINGLE-mode optical waveguide formed on a $\mathrm{Si}$ substrate has several advantages such as the possibility of integrating both optical and electrical devices, utilization of extremely clean and flat surface of an $\mathrm{Si}$ wafer, direct formation of an $\mathrm{SiO}_{2}$ cladding layer by means of thermal oxidation, easy formation of input end facet by cleavage, possibility of large scale integration, and so on. Recently some works have been reported on the formation of a single-mode waveguide on an $\mathrm{Si}$ substrate by using $\mathrm{As}_{2} \mathrm{~S}_{3}$ glass [1], SiN [2], [3], and $\mathrm{Si}_{1-x} \mathrm{Ti}_{X} \mathrm{O}_{2}$ [4], [5]. However, the problem of utilizing a thermally grown $\mathrm{SiO}_{2}$ layer is that the sufficient thickness of $\mathrm{SiO}_{2}$ cladding layer, typically more than $4 \mu \mathrm{m}$, is required to reduce the radiation loss due to the penetration of the evanescent field into the high index Si substrate $(n=3.5-3.8)$. This results in taking considerable time interval to form a thick $\mathrm{SiO}_{2}$ layer.

To solve this problem, a novel waveguide, an antiresonant reflecting optical waveguide (ARROW) has been proposed and the possibility of low loss propagation was demonstrated at a $1.3-\mu \mathrm{m}$ wavelength [6]. This novel waveguide utilizes the extremely high reflectivity of a single pair of $\mathrm{Si} / \mathrm{SiO}_{2}$ interference cladding which is about $2 \mu \mathrm{m}$ thick. However, in the near infrared and visible

Manuscript received February 20, 1987; revised November 12, 1987. This work was partially supported by the Ministry of Education, Science, and Culture under Grant-in-Aid for the Encouragement of Young Scientists 62750345, and by the Hoso Bunka Foundation, the KDD Engineering and Consulting Foundation, the Murata Science Foundation, the Okawa Institute of Information and Telecommunication, and the CASIO Science Promotion Foundation.

T. Baba and Y. Kokubun are with Yokohama National University, Faculty of Engineering, Division of Electrical and Computer Engineering, Hodogaya-ku, Yokohama, 240 Japan.

T. Sakaki was with Yokohama National University, Faculty of Engineering, Division of Electrical and Computer Engineering, Yokohama, 240 Japan. He is now with Recruit Co., Ltd., Tokyo, Japan.

K. Iga is with Tokyo Institute of Technology, Research Laboratory of Precision Machinery and Electronics, Midori-ku, Yokohama, 227 Japan.

IEEE Log Number 8822561 . wavelength range shorter than $0.9 \mu \mathrm{m}$, this waveguide is considered to suffer much loss due to the light absorption of an Si first cladding layer.

In this report, we introduce a new ARROW analysis method using a system interference matrix of thin films in order to precisely analyze its propagation characteristics. We derive a formula of optimum thickness for each cladding layer to minimize the propagation loss, and obtain approximate formulas for propagation constant and loss at the optimum thickness. Next, we describe the loss reduction of ARROW in a shorter wavelength range by employing a transparent $\mathrm{TiO}_{2}$ first cladding layer. Last we demonstrate an uncoupled stacking of two parallel ARROW's as an application of this waveguide to three-dimensional optical interconnection in waveguide devices on Si and/or LSI's.

\section{Propagation Characteristics of ARrow}

\section{A. Expression of Propagation Constant and Loss}

The structure of the ARROW is shown in Fig. 1. A core is made of $\mathrm{SiO}_{2}$. An interference cladding, which consists of high index layer named first cladding and low index $\mathrm{SiO}_{2}$ layer named second cladding, separates the core from Si substrate. Light propagates through the core by repeating total internal reflection at the upper air- $\mathrm{SiO}_{2}$ boundary and ultrahigh reflection from the interference cladding $\left(\mathrm{Si} / \mathrm{SiO}_{2}\right.$ or $\left.\mathrm{TiO}_{2} / \mathrm{SiO}_{2}\right)$ layers under the core. The reflectivity of the interference cladding is very close to unity and is slightly smaller than 1.0 , when the thicknesses of interference cladding layers satisfy the antiresonant condition. (The high reflection condition corresponds to the antiresonant state of a Fabry-Perot resonator. The name of ARROW comes from this correspondence.) Therefore, this structure is a kind of leaky waveguide, and several modes can propagate since it has no cutoff so long as the mode angle is smaller than the critical angle of the air$\mathrm{SiO}_{2}$ boundary. But higher order modes are effectively filtered out by the loss discrimination associated with the propagation angle of corresponding rays.

Let us suppose a generalized calculation model of ARROW as shown in Fig. 2. The phase condition of $\nu$ thorder mode is expressed by

$$
2 k_{0} n_{c} d_{c} \sin \theta_{\nu}+\phi_{1}+\phi_{2}=2 \pi \nu
$$

where $\phi_{1}$ and $\phi_{2}$ are the phase shifts at the interfaces between air and core and between core and interference 


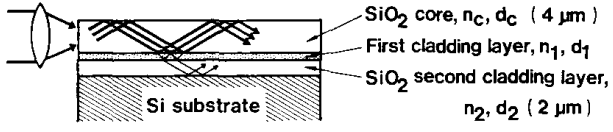

Fig. 1. Structure of ARROW.

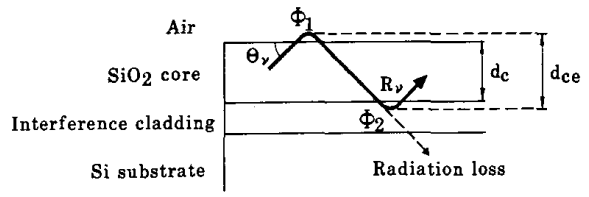

Fig. 2. Analysis model of ARROW.

cladding, respectively, $\theta_{\nu}$ is the propagation angle of light, $k_{0}$ is the wavenumber in a vacuum, and $n_{c}$ and $d_{c}$ are the refractive index and the thickness of core, respectively. Here we define an equivalent thickness of core $d_{c e}$ involving the penetration of the field into the layers outside the core as follows:

$$
d_{c e}=d_{c}+\frac{2 \pi+\phi_{1}+\phi_{2}}{2 k_{0} n_{c} \sin \theta_{\nu}} \simeq d_{c}+\zeta \frac{\lambda}{2 \pi \sqrt{n_{c}^{2}-n_{0}^{2}}}
$$

$$
\zeta= \begin{cases}1, & \text { for TE mode } \\ \left(n_{0} / n_{c}\right)^{2}, & \text { for TM mode }\end{cases}
$$

where $n_{0}$ is the refractive index of the layer upon the core (in our case air). In (2) we neglected the penetration of the field into the interference cladding from the core because it is very small when the high reflectance condition is satisfied. Equation (3) shows that $d_{c e}$ for TM mode is little smaller than that for TE mode. The propagation constant $\beta_{v}$ and loss $\alpha_{v}$ are given in terms of $\theta_{v}$ and $d_{c e}$ by

$$
\begin{aligned}
& \beta_{\nu}=k_{0} n_{c} \cos \theta_{\nu} \\
& \alpha_{\nu}=2.17\left(1-R_{\nu}\right) \tan \theta_{\nu} / d_{c e} \quad(\mathrm{~dB} / \mathrm{m})
\end{aligned}
$$

where $R_{v}$ are the power reflectivities of interference cladding layer for the $\nu$ th mode. It can easily be understood that is proportional to $\left(1-R_{\nu}\right)$ since it corresponds to the sum of powers radiated into the Si substrate and absorbed in the cladding layers.

Now we consider the fundamental mode $(\nu=0)$. The low loss condition of ARROW is that the lights reflected at individual boundaries of the cladding layers must intensify each other in the direction of the core. This is identical to the condition that the phase shift inside each cladding layer in the direction perpendicular to the surface of the substrate is $\pi / 2$. The optimum thicknesses of two layers of the interference cladding, which provide minimum loss, are given by

$$
d_{1} \simeq \frac{\lambda}{4 n_{1}}\left\{1-\left(\frac{n_{c}}{n_{1}}\right)^{2}+\left(\frac{\lambda}{4 n_{1} d_{c e}}\right)^{2}\right\}^{-1 / 2}(2 N+1)
$$

$$
d_{2} \simeq \frac{d_{c e}}{2}(2 M+1) \quad(N, M=0,1,2, \cdots) .
$$

When $d_{1}$ and $d_{2}$ are optimum and all layers are transparent, the propagation constant and minimum loss of fundamental mode $(\nu=0)$ is approximately expressed by

$$
\begin{aligned}
\beta_{0}= & k_{0} n_{c}\left\{1-\left(\frac{\lambda}{4 n_{c} d_{c e}}\right)^{2}\right\}^{1 / 2} \\
\alpha_{0}= & X \frac{5428.68 \lambda^{4}}{n_{c} d_{c e}^{5}\left\{1-\left(\frac{\lambda}{4 n_{c} d_{c e}}\right)^{2}\right\}^{1 / 2}} \\
& \cdot 1 /\left\{n_{1}^{2}-n_{c}^{2}+\left(\frac{\lambda}{4 d_{c e}}\right)^{2}\right\} \\
& \cdot 1 /\left\{n_{s}^{2}-n_{c}^{2}+\left(\frac{\lambda}{4 d_{c e}}\right)^{2}\right\}^{1 / 2} \quad(\mathrm{~dB} / \mathrm{cm}) \\
X= & \begin{cases}1, & \text { for TE mode } \\
\left(n_{1}^{2} n_{s} / n_{c}^{3}\right)^{2}, & \text { for TM mode }\end{cases}
\end{aligned}
$$

where the units of $\lambda$ and $d_{c e}$ are in micrometers. Equation (9) shows that $\alpha_{0}$ depends greatly on $\lambda$ and $d_{c e}$, that is, the smaller $\lambda$ is and the larger $d_{c e}$ is, the smaller $\alpha_{0}$ is. Likewise, the larger the differences of refractive indices $n_{1}^{2}-n_{c}^{2}$ and $n_{s}^{2}-n_{c}^{2}$ provide, the smaller $\alpha_{0}$ is. Equation (10) shows that the loss discrepancy between TE and TM modes is due to the large difference of refractive indices between $\mathrm{SiO}_{2}$ core and the first cladding layer, and also between the core and the substrate.

In contrast with this, as easily seen from (6) and (7), the loss of first higher order mode $(\nu=1)$ is very high when $d_{1}$ and $d_{2}$ are optimum for the fundamental mode. However the loss of second higher order mode $(\nu=2)$ can be low to some extent. The loss $\alpha_{2}$ is expressed approximately in terms of $\alpha_{0}$ independently of wavelength and the waveguide parameters by

$$
\alpha_{2} \simeq 81 \alpha_{0} \text {. }
$$

\section{B. Mode Analysis of ARROW by System Interference Matrix}

Next, we simulated the propagation characteristics of ARROW by calculating the phase shifts $\phi_{1}$ and $\phi_{2}$ and the power reflectivities $R_{1}$ and $R_{2} . \phi_{1}, \phi_{2}, R_{1}$, and $R_{2}$ are expressed in terms of the electromagnetic field reflectivity $r_{1}$ and $r_{2}$ by

$$
\left.\begin{array}{l}
\phi_{l}=-\arg \left(r_{l}\right) \\
R_{l}=\left|r_{l}\right|^{2}
\end{array}\right\} \quad(l=1,2) .
$$

The first reflectivities $r_{1}$ and $r_{2}$ are calculated from the optical thin film theory which is often used for multilayer interference filters and expressed by

$$
r_{l}=\frac{m_{11}-Y_{s} Y_{c}^{-1} m_{22}+Y_{s} m_{12}-Y_{c}^{-1} m_{21}}{m_{11}+Y_{s} Y_{c}^{-1} m_{22}+Y_{s} m_{12}+Y_{c}^{-1} m_{21}}
$$


where $Y_{c}$ and $Y_{s}$ are the admittances of the core and the substrate, respectively. $m_{11}-m_{22}$ are the components of system interference matrix $M$ which are given by

$M=\left[\begin{array}{ll}m_{11} & m_{12} \\ m_{21} & m_{22}\end{array}\right]=\prod_{i}\left[\begin{array}{ll}\cos \varphi_{i} & j Y_{i}^{-1} \sin \varphi_{i} \\ j Y_{i} \sin \varphi_{i} & \cos \varphi_{i}\end{array}\right]$.

Here $\varphi_{i}$ is the complex phase shift including the absorption, and $Y_{i}$ is the admittance in the $i$ th layer. These are expressed by

$$
\begin{aligned}
& \varphi_{i}=k_{0} n_{i} d_{i} \sin \theta_{i} \\
& Y_{i}= \begin{cases}-n_{i} \sin \theta_{i}, & \text { for TE mode } \\
n_{i} / \sin \theta_{i}, & \text { for TM mode }\end{cases}
\end{aligned}
$$

where $n_{i}$ and $d_{i}$ are the complex refractive index and thickness of the $i$ th layer, respectively, and the $\theta_{i}$ is the angle obtained from $\theta_{\nu}$ by Snell's low. $\theta_{i}$ takes a complex value when layers include the absorption and/or in the case of total internal reflection.

The procedure of the numerical calculations is as follows: First, we assume a definite value of $\theta_{\nu}$ and calculate $\phi_{1}, \phi_{2}, R_{1}$ and $R_{2}$ by using (12)-(17). Next, we judge whether they satisfy the phase condition of (1) or not. If they do not, we try again by changing the value of $\theta_{\nu}$ little by little until they satisfy the phase condition. Repeating this process, we can reach the propagation angle $\theta_{\nu}$ which leads the propagation constant and the loss through (4) and (5).

Fig. 3 shows the calculated power reflectivitly $R$ of $\mathrm{TiO}_{2} / \mathrm{SiO}_{2}$ interference reflector versus the incident angle of light at $\lambda=0.633 \mu \mathrm{m}$ when the thickness of $\mathrm{TiO}_{2}$ first cladding layer is $0.088 \mu \mathrm{m}$ and that of $\mathrm{SiO}_{2}$ second cladding layer is $2 \mu \mathrm{m}$. These thicknesses satisfy the condition of (6) and (7) when the thickness of core is $4 \mu \mathrm{m}$. The vertical axis is $(1-R)$ which is scaled in a logarithm, since it is directly related to the propagation loss through (4). Three arrows indicate the first three propagation angles of ARROW which satisfy the phase condition for TE modes given by (1) when $d_{c}=4 \mu \mathrm{m}$. Although the reflector consists of only two layers, its reflectivity for the TE fundamental mode is extremely high ( 99.98 percent). In comparison with this, the reflectivities of second and third order modes are fairly low. The reflectivity of TM mode is much lower than TE mode all over the range of angle since there exists a Brewstar angle around $20^{\circ}$.

Fig. 4 shows the calculated TE mode loss of the ARROW at $\lambda=0.633 \mu \mathrm{m}$. Fig. 4(a) shows the TE mode loss characteristics versus the thickness of first cladding layer $d_{1}$ when $d_{c}=4 \mu \mathrm{m}$ and $d_{2}=2 \mu \mathrm{m}$. If an a-Si is used as the first cladding layer, of which the refractive index is $n=4.2+j 0.30$, the guided light suffers a 2.76 $\mathrm{dB} / \mathrm{cm}$ loss at the optimum value of $d_{1}=0.039 \mu \mathrm{m}$ owing a large absorption of $\mathrm{Si}$ at this wavelength. On the other hand, the loss can be considerably reduced if a transparent material such as $\mathrm{TiO}_{2}, \mathrm{GeO}_{2}, \mathrm{Si}_{3} \mathrm{~N}_{4}, \mathrm{Al}_{2} \mathrm{O}_{3}$, etc., whose refractive index is higher than that of $\mathrm{SiO}_{2}$,



Fig. 3. Reflectivity of $\mathrm{TiO}_{2} / \mathrm{SiO}_{2}$ interference reflector versus incident angle.

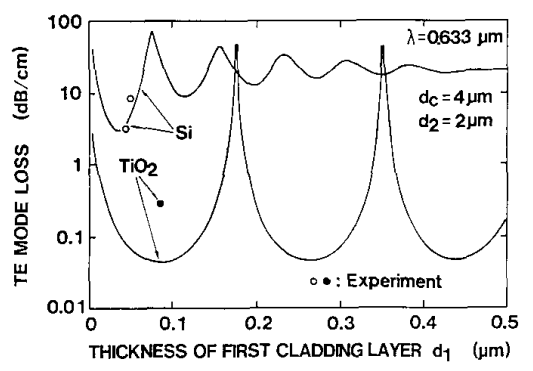

(a)

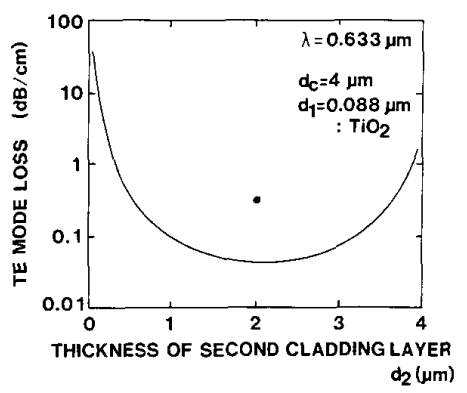

(b)

Fig. 4. TE mode loss characteristics of ARROW. (a) TE mode loss versus thickness of first cladding layer. (b) TE mode loss versus thickness of second cladding layer.

is employed. If $\mathrm{TiO}_{2}(n=2.3)$ is adopted, for example, the loss of the fundamental mode is calculated as 0.046 $\mathrm{dB} / \mathrm{cm}$ from (9), when $d_{1}=0.088 \mu \mathrm{m}$. Fig. 4(b) shows the TE mode loss characteristics versus the thickness of second cladding layer, when the first cladding layer is $\mathrm{TiO}_{2}$ with $0.088-\mu \mathrm{m}$ thickness, and $d_{c}=4 \mu \mathrm{m}$. The loss reaches minimum at $d_{2}=2 \mu \mathrm{m}$. It can be seen from these figures that the tolerance of the thicknesses $d_{1}$ and $d_{2}$ is fairly large. This implies that the low-loss wavelength region is also broad. In contrast with this the loss of the first and second higher order modes are larger about 55.0 $\mathrm{dB} / \mathrm{cm}$ and $3.74 \mathrm{~dB} / \mathrm{cm}$, respectively. This can be understood from Fig. 3 and equation (5). Fig. 5(a) and (b) illustrates the transverse electromagnetic field profiles of the TE and TM fundamental modes, respectively. The TE mode field is confined strongly inside the core and little light radiates to the substrate (Fig. 5(a)). On the other hand, the radiation of the TM mode field is large since 


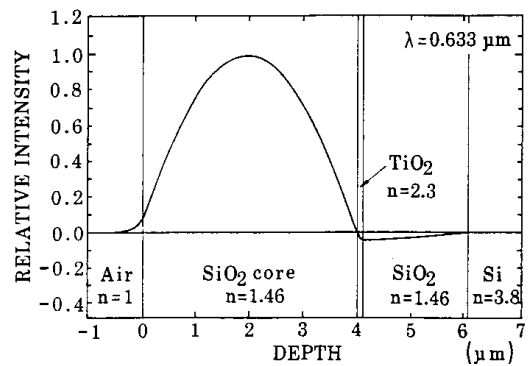

(a)

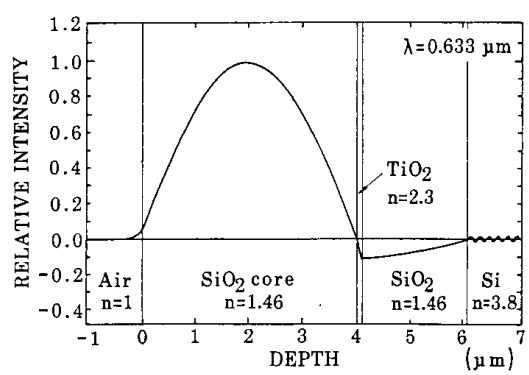

(b)

Fig. 5. Field profile of ARROW. (a) TE fundamental mode. (b) TM fundamental mode.

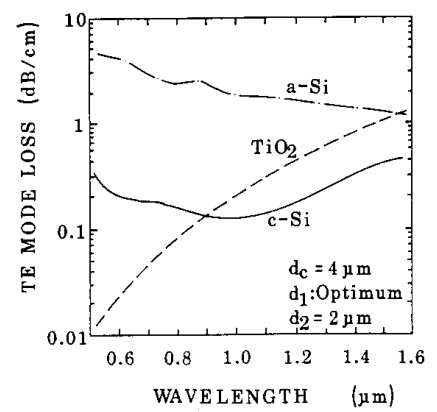

(b)

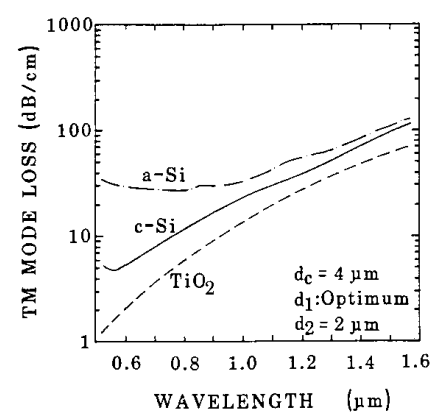

(a)

Fig. 6. Minimum loss characteristics versus wavelength. (a) TE mode. (b) TM mode.

the differential of the field is discontinuous at the interface of each layer (Fig. 5(b)).

Fig. 6 shows the minimum loss of ARROW against the wavelength. The variations of refractive index and $a b-$ sorption of each layer against the wavelength are taken into account when a-Si, c-Si, and $\mathrm{TiO}_{2}$ are adopted for the first cladding layer [9]. It can be seen that if $\mathrm{TiO}_{2}$ is used, the $\mathrm{TE}_{0}$ mode loss is low enough in the range shorter than $0.9 \mu \mathrm{m}$, but that it increases gradually corresponding with (9) in the wavelength range longer than $0.9 \mu \mathrm{m}$. Poly-Si, whose refractive index is larger than $\mathrm{TiO}_{2}$ and is transparent, is more efficient to obtain a low-loss ARROW in this wavelength range. The loss of TM mode is far larger than that of TE mode in the whole wavelength range.

\section{Fabrication and Measurement}

The ARROW was fabricated by RF sputtering for $\mathrm{SiO}_{2}$ second cladding and core layers and by electron beam evaporation for the $\mathrm{TiO}_{2}$ first cladding layer. The loss of several samples was measured by cleaving the waveguide into various lengths and measuring the insertion loss as a function of waveguide length.

Results are also shown in Fig. 7 (also shown in Fig. 4 by closed and open circles). In this figure, the inclination of each line corresponds to the loss per unit length and the intercept corresponds to the coupling loss at the input end. The measured loss of Si first cladding layer was 3.0 $\mathrm{dB} / \mathrm{cm}$ when $d_{1}$ was $0.049 \mu \mathrm{m}$. This result almost coincides with the theoretical value as shown in Fig. 4. In contrast with this, the loss of $\mathrm{TiO}_{2}$ first cladding was as low as $0.3 \mathrm{~dB} / \mathrm{cm}$ when $d_{1}$ was $0.08 \mu \mathrm{m}$. The discrepancy between measured and theoretical values seems to be caused by the light scattering due to the surface roughness of the core. A single mode propagation was confirmed from the near field pattern of the output light from $\mathrm{TiO}_{2} / \mathrm{SiO}_{2}$ ARROW as shown in Fig. 8 .

\section{Uncoupled Stacking of ARROW's}

In order to achieve a very large scale integration of optical waveguide devices, it will be necessary to construct three-dimensional optical interconnection, for example, by stacking independent optical waveguides. If we try to stack conventional dielectric waveguides, however, the cladding layer must be thick enough to maintain the isolation between those waveguides. As mentioned above, ARROW can confine the optical field into the core strongly with only $2-\mu \mathrm{m}$-thick interference cladding. Therefore we can stack this waveguide on another waveguide with only $2-\mu \mathrm{m}$ spacing without any coupling between them.

In Fig. 9 we show the structure of stacked ARROW's. Light propagates through two $\mathrm{SiO}_{2}$ cores of $4 \mu \mathrm{m}$ thickness. The cores are separated by $\mathrm{TiO}_{2} / \mathrm{SiO}_{2} / \mathrm{TiO}_{2}$ intermediate cladding. The light cannot propagate through the intermediate $\mathrm{SiO}_{2}$ layer of $2-\mu \mathrm{m}$ thickness between cores. This is because if we regard this layer as a core, $4-\mu \mathrm{m}$ second cladding is just the even multiple of the half of core thickness $(2 \mu \mathrm{m})$ and satisfies a low reflection condition, which can be seen from (5).

The stacked ARROW's were fabricated by repeating the 


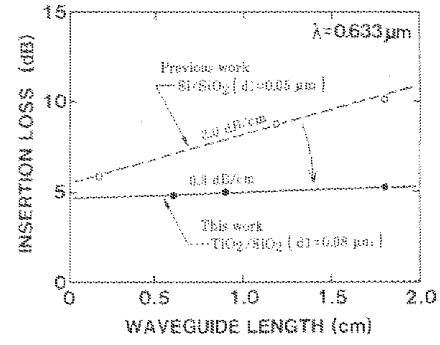

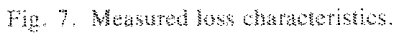

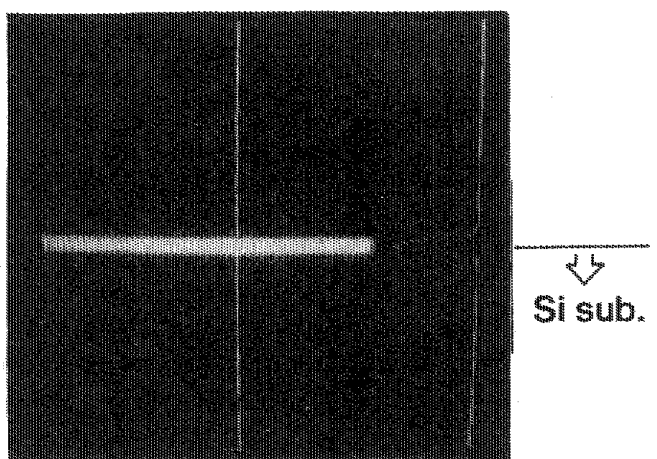

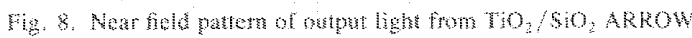

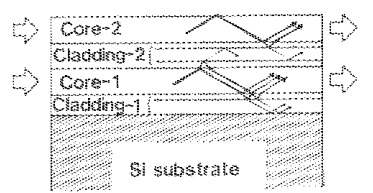

Fig. 5. Srucure of stacked AREOY's

same proess as mentioned in Section II. The deaved end of the stacked wagude and the thicknesses of lay. ers are shown in Fig. 10. Fig. II shows the near held pattems (NFP's) of output light. Fig. 1 (a) show the NFP when both waveguides were illuminated simultuneously by defocusing the position of the focused beam at the input end. Very lituelight is observed from the intemediate 510 cladding of 2 mm thickness. Fig. $11(b)$ and (c) shows the NFP's when the light was coupled into cither upper or lower waveguide, respectively. Most of the light was guided through only the exiled waveguide, except that a small amoun of scattered light hom upper waveguide was observed in Fig. $11(\mathrm{c})$.

The propagation losses were measured independently by a method similar to the one mentioned in section II. The resuls were $9.8 \mathrm{~dB} / \mathrm{cm}$ for waveguide 2 (upper core) and $4.5 \mathrm{~dB} / \mathrm{cm}$ for waveguide 1 (lower core). These are larger than the theoretical values $(\alpha<0.052 \mathrm{~dB} / \mathrm{cm})$. This discrepency seens to be caused manly by the light scattering due to the accuntuation of the surface roughness of each layer. Reduction of the loss will be possble

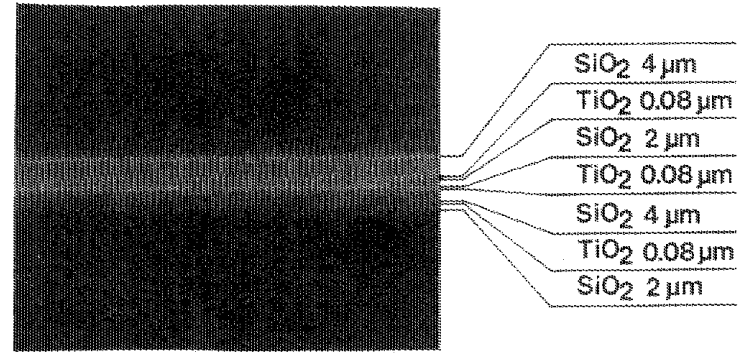

Fig. I0. Cleaved an hase of stacked ARROW'

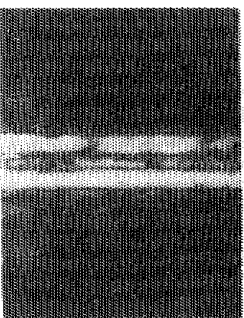

(a)

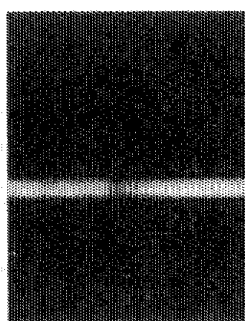

(b)

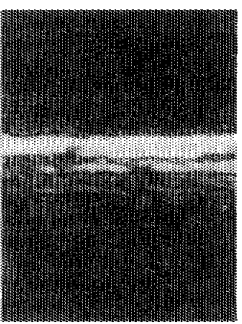

(c)

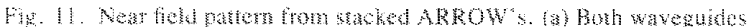

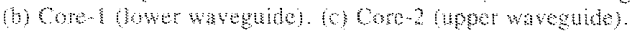

by mproving the fabrication process and reducing the surtace contamintion.

\section{Concuusion}

In this paper, we have deseribed the optimum design and loss reduction of ARROW. First, we introduced a new andysis method of optical wavegude induding a loaky type such as ARROW. This method wilizes the system interterence matrix well known for multayer optical thin fims. Using this nethod, we andyzed the los character. istics of ARROW in the wavelength range shorter than $0.9 \mathrm{~km}$. We obtaned the low loss condtions of thicknesses of interference clading layers and denived approximate expressions for propagation constant and the loss, which are consistent well wh the precise numerical calculation. Next, we fabricated the ARROW by using transparent $\mathrm{TO}_{2} / \mathrm{SO}_{2}$ interference cladding and the loss was renakably reduced down to $0.3 \mathrm{~dB} / \mathrm{cm} a=0.633$ m. Then wo ARROW's were sacked and the uncoupled light propagation through each wavguide was realized with only 2 -um spacing. This technque will provide a vrety of independent optcal interconnections in the verhical drecion.

\section{ACKNOWHOMGEN}

The athors wold like to than Prof. M. Toki and Prof. Y. Turuki, Yokohma National University, and Prot. Y. Suematsu. Tokyo Instute of Technology, for support. They hank T. Sakaguch, Tokyo Instrute of Technology. for help with the experiment. The wuthors diso wish to thank Dr. H. Nakajima and Dr. M. Semo. Fijisu Laboratories Ltd., for heir supply of silicon walers. 


\section{REFERENCES}

[1] T. Suhara, Y. Handa, H. Nishihara, and J. Koyama, "Monolithic integrated micrograting and photodiodes for wavelength demultiplexing," Appl. Phys. Lett., vol. 40, p. 120, 1982.

[2] W. Stutius and W. Streifer, "Silicon nitride films on silicon for optical waveguides,' Appl. Opt., vol. 16, p. 3218, 1977.

[3] D. E. Zelmon, J. T. Boyd, and H. E. Jackson, "Low loss optical waveguides fabrication by thermal nitridation of oxidized silicon," Appl. Phys. Lett., vol. 47, p. 353, 1985

[4] M. Seino, M. Sibayama, I. Sawaki, and H. Nakajima, " $\mathrm{Si}_{1}-x \mathrm{Ti}_{X} \mathrm{O}_{2}$ guided-wave connecting circuits grown by magnetron sputtering," presented at 8th Conf. on Optical Fiber Communications, San Diego, CA, 1985, pap. WK5.

[5] N. Takato, M. Yasu, and M. Kawachi, "Low-loss high-silica singlemode channel waveguides," Electron. Lett., vol. 22, p. 321, 1986.

[6] M. A. Duguay, Y. Kokubun, T. L. Koch, and L. Pfeiffer, "Antiresonant reflecting optical waveguides in $\mathrm{SiO}_{2}-\mathrm{Si}$ multi-layer structures," Appl. Phys. Lett., vol. 49, p. 13, 1986.

[7] Z. Knittl, "Optics of thin films,"' in An Optical Multilayer Theory. New York: Wiley-Interscience, 1976.

[8] Y. Kokubun, T. Baba, T. Sakaki, and K. Iga, "Low loss antiresonant reflecting optical waveguide on Si substrate in visible wavelength region," Electron. Lett., vol. 22, p. 892, 1986.

[9] E. D. Palik, Handbook of Optical Constants of Solids. New York: Academic, 1985

[10] Y. Kokubun, T. Baba, and K. Iga, "Uncoupled stacking of parallel ARROW's for three-dimensional optical wiring," presented at 12th Euro. Conf. on Optical Communication, Barcelona, Spain, 1986, pap. Tu-C3

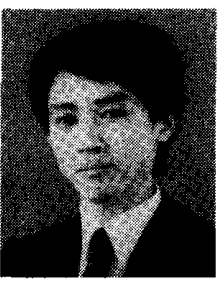

Toshihiko Baba was born in Ueda City, Negano Prefecture, Japan, on November 12, 1962. He received the B.E. degree in electrical engineering and M.E. degree in electrical and computer engineering from the Yokohama National University, Japan, in 1985 and 1987 , respectively. He is currently working on towards the Dr.Eng. degree at the Graduate School of Yokohama Nationa University.

Mr. Baba is a member of the Institute of Electronics, Information, and Communication Engineers and of the Japan Society of Applied Physics.

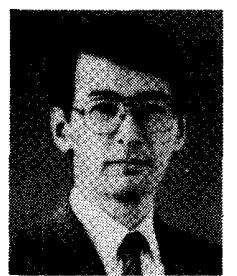

Yasuo Kokubun (M'85) was born in Fukushim Prefecture, Japan, on July 7,1952 . He received the B.E. degree from Yokohama National University, Yokohama, Japan, in 1975, and the M.E. and Ph.D. degrees from Tokyo Institute of Technology, Tokyo, Japan, in 1977 and 1980, respectively.

After he worked for the Research Laboratory of Precision Machinery and Electronics, Tokyo Institute of Technology, as an Research Associate from 1980 to 1983, he joined the Yokohama Na- tional University as an Associate Professor in 1983. His current research is in integrated optics, especially waveguide type optical devices. From 1984 to 1985 he was with Bell Laboratories, Holmdel, NJ, as a Member of the Technical Staff and was engaged in research on a novel waveguide on a semiconductor substrate (ARROW) for integrated optics. He is the coauthor of Fundamentals of Microoptics (Academic, 1984) and Optical Fiber (Ohm-sha 1986).

Dr. Kokubun is a member of the Institute of Electronics, Information, and Communication Engineers, the Japan Society of Applied Physics, and the Optical Society of America.

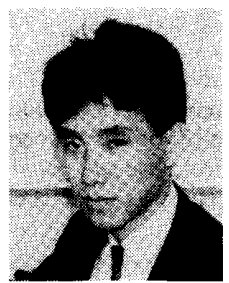

Toshihito Sakaki was born in Kagoshima City, Kagoshima Prefecture, Japan, on March 23, 1963 $\mathrm{He}$ received the B.E. degree in electrical engineering from the Yokohama National University, Japan, in 1985 .

He is now with the Recruit Co., Ltd., Tokyo, Japan.



Kenichi Iga (S'67-M'68-SM'80-F'87) was born in Hiroshima Prefecture, Japan, on June 15, 1940. He received the B.E., M.E., and Ph.D. degrees from Tokyo Institute of Technology, Tokyo, Japan, in 1963, 1965, and 1968, respectively.

He joined the Research Laboratory of Precision Machinery and Electronics, Tokyo Institute of Technology, as a Research Associate in 1968 , and became an Associate Professor in 1973, and a Professor in 1984. He is also responsible for education in the Graduate School at Nagatuta Campus, Tokyo Institute of Technology, as a Concurrent Professor. He is presently engaged in research on optical fiber communications, surface-emitting semiconductor lasers, integrated optics and microoptic systems including large-scale optics (LSO). From 1979 to 1980 he was with Bell Laboratories, Holmdel, NJ, as a Visiting Technical Staff Member and was engaged in GaInAsP/InP technology for single-mode lasers and integrated optics. $\mathrm{He}$ is the author of Fundamentals of Microoptics (Academic, 1984), Fundamentals of Laser Optics (Ohm-sha), and several other books.

Dr. Iga is a member of the Institute of Electronics, Information, and Communication Engineers, the Japan Society of Applied Physics, and the Optical Society of America. He received the Inada Memorial Prize in 1966, the Book Award for Introduction to Optical Fiber Communications in 1978, and the Paper Award in 1986 from the Institute of Electrical, Information, and Communication Engineers. 